\title{
Silver Nanoparticle Impregnated Porous Carbon as a Nonenzymatic Hydrogen Peroxide Sensor
}

\author{
Yi-Song Zou ${ }^{1+}$, Miao-Miao Jia ${ }^{1+}$, Jun-Qin Fan ${ }^{1}$, Fa-Mei Qin ${ }^{1}$, Jian-Guo Yu ${ }^{1 *}$, Yong-Nan Zhao ${ }^{2}$ \\ ${ }^{1}$ College of Environment and Chemical Engineering \& State Key Laboratory of Hollow-Fiber \\ Membrane Materials and Membrane Processes, Tianjin Polytechnic University, Tianjin, P.R. China \\ ${ }^{2}$ School of Materials Science and Engineering, Tianjin Polytechnic University, Tianjin, P.R. China \\ *E-mail: hh_y1118@hotmail.com \\ the authors contribute equally to this work.
}

doi: $10.20964 / 2016.07 .04$

Received: 14 March 2016 / Accepted: 3 May2016 / Published: 4 June 2016

In this work, a non-enzymatic hydrogen peroxide sensor has successfully been realized based on $\mathrm{Ag}$ nanoparticles doped carbon modified carbon paste electrode (AgNPs/C-CPE) in $\mathrm{pH}=7$ phosphate buffer solution (PBS). The Ag nanoparticles doped carbon composites (AgNPs/C) were synthesized by a simple one-step carbonization method using sucrose with $\mathrm{AgNO}_{3}$ as the carbon precursor. AgNPs/CCPE for electrochemical responds of hydrogen peroxide was explored by using cyclic voltammetry (CV) and amperometry. Besides, contents of AgNPs and $\mathrm{pH}$ values were optimized. The AgNPs/CCPE containing $13.5 \mathrm{wt} \%$ AgNPs exhibits the highest electrocatalytic activity for the detection of hydrogen peroxide in $\mathrm{pH}=7 \mathrm{PBS}$. At an applied potential of $0 \mathrm{~V}$, the non-enzymatic sensor of hydrogen peroxide also showed a good linear relationship in a wide concentration range of 0.5-400 $\mu \mathrm{M}$ with a correlation coefficient of 0.9987 , a detection limit of $0.3 \mu \mathrm{M}$ at a signal-to noise ratio of 3 , and a high sensitivity of $309.4 \mu \mathrm{A} \mathrm{cm} \mathrm{cm}^{-1}$. The amorphous carbon-supported AgNPs not only avoided the use of chemical reductants but also improved the electrocatalytic activity and stability of Ag NPs.

Keywords: Nano-silver, hydrogen peroxide determination, nonenzymatic sensor, sucrose, electrocatalysis

\section{FULL TEXT}

(C) 2016 The Authors. Published by ESG (www.electrochemsci.org). This article is an open access article distributed under the terms and conditions of the Creative Commons Attribution license (http://creativecommons.org/licenses/by/4.0/). 